\title{
Worldvolume uncertainty relations for D-branes
}

\author{
Chong-Sun Chu \\ Institute of Physics, University of Neuchâtel, CH-2000 Neuchâtel, Switzerland \\ Pei-Ming Ho and Yeong-Chuan Kao \\ Department of Physics, National Taiwan University, Taipei 10764, Republic of China \\ (Received 10 May 1999; published 15 November 1999)
}

\begin{abstract}
By quantizing an open string ending on a D-brane in a nontrivial supergravity background, we argue that there is a new kind of uncertainty relation on a D-brane worldvolume. Furthermore, we fix the form of the uncertainty relations and their dependence on the string coupling constant by requiring them to be consistent with various string theory and $\mathrm{M}$ theory dualities. In this way we find a web of uncertainties of spacetime for all kinds of brane probes, including fundamental strings, D-branes of all dimensions as well as M theory membranes and fivebranes. [S0556-2821(99)06522-4]

PACS number(s): 11.25.Mj, 11.25.Sq
\end{abstract}

\section{INTRODUCTION}

String theory is a promising candidate of quantum gravity and the theory of spacetime. It is therefore an interesting question to study the properties of spacetime in string theory. A number of uncertainty relations have been proposed in relation to string theory. See, for example, $[1,2]$ for a review of the subject and for further references. It was first proposed [3] that a modified version of the canonical Heisenberg uncertainty relation,

$$
\delta X \delta P \geqslant 1+l_{s}^{2} \delta P^{2},
$$

governs the high energy behavior of string theory. This relation implies the existence of a minimal scale $\delta X \sim l_{s}$ [3]. Another uncertainty relation is the one proposed by Yoneya [4]:

$$
\delta T \delta X \geqslant l_{s}^{2} .
$$

He further suggested that the spacetime uncertainty relation can be one of the fundamental principles underlying nonperturbative string theory, and can also be one of the guiding principles for constructing a covariant formulation of $\mathrm{M}$ theory [4].

In this paper, we propose a new kind of uncertainty relation:

$$
\delta X^{0} \delta X^{1} \ldots \delta X^{p} \geqslant g_{s} l_{s}^{p+1}
$$

for the worldvolume of a $\mathrm{D} p$-brane. Here $X^{i}$ are the D-brane worldvolume coordinates. We also propose a similar kind of uncertainty relation for M-branes.

It is well known that in string theory different probes could see slightly different spacetime geometries. So it is natural to expect that the uncertainty principle can be different for different probes, as is manifest in our proposed relations (3). Note that Eq. (3) is involved only with the longitudinal directions on a D-brane, that is, the D-brane worldvolume. Another uncertainty relation involving both longitudinal and transverse directions for a D-brane was proposed by Yoneya and collaborators $[2,4,5]$. He proposed that if one interprets $T$ and $X$ as the longitudinal and transverse coordinates of a D-brane, Eq. (2) can also be understood as an uncertainty relation for a D-brane.

In the brane world scenario [6], it is argued that we could be living on a D-brane. It is thus of great interest to determine the form of uncertainty relations on a D-brane worldvolume, which will be interpreted as the uncertainty relation for the four dimensional spacetime on which we are trapped. Since it is also known that a D-brane worldvolume becomes noncommutative $[7,8]$ in the presence of a nontrivial background $B_{N S-N S}$ field in the direction of the brane, it is natural to ask what kind of uncertainty relation follows this worldvolume noncommutativity $[9,10]$. In this paper we first show how to obtain the uncertainty relation for a D1-brane by integrating out quantum fluctuations of the background field. Then we use various dualities in string theory and $M$ theory to extend the uncertainty relation to other D-branes and strings or membranes. Putting all the old and new dualities together, we find a web of spacetime uncertainty relations associated with all the branes in string theory or M theory.

The organization of this paper is as follows. In Sec. II, we first extend the previous results $[7,8,10]$ about the noncommutativity of D-brane by generalizing the background to the first nontrivial (yet manageable) order, with a curved background metric $g_{\mu \nu}$ and a nontrivial Neveu-Schwarz-NeveuSchwarz (NS-NS) 2-form field. Then we argue in Sec. III that these commutation relations give rise to uncertainty relations on the D-brane worldvolume. While the precise form of the uncertainty relations cannot be easily fixed this way, one can use the dualities of string theory and $\mathrm{M}$ theory to constrain the form of the uncertainty relations. Guided by this idea, we propose in Sec. IV worldvolume uncertainty relations for D-branes, M2-branes, M5-branes, as well as fundamental strings. We check that they are consistent with various dualities of string theory and M theory. Finally we make a few remarks in Sec. V.

\section{NONCOMMUTATIVITY FROM OPEN STRING QUANTIZATION}

It was first proposed by Connes, Douglas, and Schwarz [7], and by Douglas and Hull [8], that the matrix model 
compactified on a torus with the NS-NS $B$ field background should be described by a field theory living on a noncommutative space. This conjecture has been verified in various ways (see, e.g., [11]). In particular, it was pointed out in [12] that this can be seen by quantizing an open string ending on a D-brane. Based on the intimate relation between compactified matrix models and D-brane worldvolume theories, Hauffman and Verlinde [13] also suggested that the low energy effective theory for a D-brane compactified on a torus with $B$ field background should also live on a noncommutative space. Following [12], the quantization of the open string ending on a D-brane with a constant $\mathcal{F}=B-F$ field background in flat spacetime was carried out in [10]. An agreement with previous works was obtained but the framework and results of [10] were more general. In this section we further generalize the previous work to a curved background and nonconstant $\mathcal{F}$ field. The derivation is in parallel with [10].

For an open string with an endpoint sticking to a D $p$-brane with $U(1)$ field strength $F$ in a $B$-field background, the bosonic part of its action can be written as

$S_{B}=\frac{1}{4 \pi \alpha^{\prime}} \int_{\Sigma} d^{2} \sigma\left[\eta^{\alpha \beta} g_{\mu \nu} \partial_{\alpha} X^{\mu} \partial_{\beta} X^{\nu}+\epsilon^{\alpha \beta} \mathcal{F}_{\mu \nu} \partial_{\alpha} X^{\mu} \partial_{\beta} X^{\nu}\right]$,

where

$$
\mathcal{F}=B-F
$$

is the modified Born-Infeld field strength on the D-brane, $F$ $=d A$, and $A_{i}, i=0,1, \ldots, p$, is the $U(1)$ gauge potential living on the $\mathrm{D} p$-brane. We use the convention $\eta^{\alpha \beta}$ $=\operatorname{diag}(-1,1)$ and $\epsilon^{01}=1$ as in [10]. Note that $\mathcal{F}$ is invariant under the gauge transformations

$$
A \rightarrow A+d \Lambda, \quad B \rightarrow B,
$$

and

$$
B \rightarrow B+d \Lambda, \quad A \rightarrow A+\Lambda .
$$

The equations of motion are

$$
\begin{gathered}
g_{\mu \kappa}\left(\ddot{X}^{\kappa}+\Gamma_{\mu \nu}^{\kappa} \dot{X}^{\mu} \dot{X}^{\nu}\right)-g_{\mu \kappa}\left(X^{\prime \kappa}+\Gamma_{\mu \nu}^{\kappa} X^{\prime \mu} X^{\prime \nu}\right) \\
+H_{\mu \nu \lambda} \dot{X}^{\nu} X^{\prime \lambda}=0
\end{gathered}
$$

where

$$
\Gamma_{\mu \nu}^{\lambda}=\frac{1}{2} g^{\lambda \kappa}\left(\partial_{\mu} g_{\kappa \nu}+\partial_{\nu} g_{\mu \kappa}-\partial_{\kappa} g_{\mu \nu}\right)
$$

is the Riemannian connection for the metric and

$$
H_{\mu \nu \lambda}=\partial_{\mu} \mathcal{F}_{\nu \lambda}+\partial_{\nu} \mathcal{F}_{\lambda \mu}+\partial_{\lambda} \mathcal{F}_{\mu \nu},
$$

that is, $H=d \mathcal{F}$. In fact, since $d F=0, H=d B$. The boundary conditions are

$$
X^{\prime \nu} g_{\nu i}+\dot{X}^{\nu} \mathcal{F}_{\nu i}=0 \text { for } i=0,1, \ldots, p,
$$

$$
X^{a}=x_{0}^{a} \text { for } a=p+1, \ldots, 9,
$$

at $\sigma=0, \pi$, where $x_{0}^{a}$ gives the position of the $\mathrm{D} p$-brane.

For convenience we have chosen the spacetime coordinates in such a way that $x_{0}^{a}$ are constant on the $\mathrm{D} p$-brane. We will use the indices $(i, j, \ldots)$ for directions parallel to the brane $(i=0,1, \ldots, p)$ and the indices $(a, b, \ldots)$ for directions transverse to the brane $(a=p+1, \ldots, 9)$. We will choose the coordinates such that $g_{i a}=0$ on the D-brane for convenience. The momentum density is

$$
2 \pi \alpha^{\prime} P_{\mu}=\dot{X}^{\nu} g_{\nu \mu}+X^{\prime}{ }^{\nu} \mathcal{F}_{\nu \mu} .
$$

For a constant background ( $\mathcal{F}=$ constant $)$ in a flat spacetime $\left(g_{\mu \nu}=\eta_{\mu \nu}\right)$, one can solve the equations of motion and boundary conditions exactly and carry out the canonical quantization [10]. The final result is

$$
\left[X^{i}, X^{j}\right]= \pm 2 \pi i \alpha^{\prime}\left(M^{-1} \mathcal{F}\right)^{i j},
$$

where

$$
M_{i j}=\eta_{i j}-\mathcal{F}_{i}{ }^{k} \mathcal{F}_{k j},
$$

and $X^{i}$ is the spacetime coordinates of the open string at the two end points $\sigma=0, \pi$. The indices are lowered or raised by the spacetime metric $\eta$. While this result does not need a compactification, in case the spacetime is compactified on a torus, the right hand side of Eq. (14) for $F=0$ is proportional to the dual $B$ field on the dual torus. It agrees with the results for the Banks-Fischler-Shenker-Susskind (BFSS) matrix model [7]. For the case of a non-constant $\mathcal{F}$ on a flat torus and its relation to deformation quantization, see [14].

For a generic background, we are unable to find the most general solution in parallel of Eq. (14). However it is possible to find a special solution in a certain approximation of weak field and slow variation. Consider a deviation from the trivial background $\mathcal{F}=0, g=\eta$ with small $\mathcal{F}$ and $\partial g$ considered as first order quantities. We will consider $\partial \partial g$ as second order and so from the Einstein equation for the background, $\partial \mathcal{F} \sim \partial g$ are also of the first order. In summary, we will consider $(x, \dot{x}, g)$ as terms of the 0th order, $(\mathcal{F}, \partial \mathcal{F}, \partial g)$ as terms of the first order, and $\left(\partial^{n} \mathcal{F}, \partial^{n} g\right), n \geqslant 2$ as terms of higher orders. We will only keep terms of the 0th and first order consistently. To solve the equations of motion and boundary conditions, we use the following ansatz for $X$ :

$$
X^{i}=x^{i}(\tau)+y^{i}(\tau) \sigma, \quad X^{a}=x_{0}^{a} .
$$

It is easy to check that this ansatz for the $\sigma$ dependence is consistent with our approximation. Substituting Eq. (16) into the boundary conditions (11), one finds that $y$ is of first order:

$$
y^{i}=-\dot{x}^{j}\left(\mathcal{F} g^{-1}(x)\right)_{j}{ }^{i} .
$$

For this ansatz, the equations of motion (8) give the geodesic equation for $x^{i}$,

$$
\ddot{x}^{i}+\Gamma(x)_{j k}^{i} \dot{x}^{j} \dot{x}^{k}=0,
$$


and the free motion for $y$ :

$$
\ddot{y}^{i}=0 .
$$

Eq. (19) is satisfied by Eq. (17) up to second order terms.

The lowest energy modes on a string are thus approximately given by

$$
\begin{gathered}
X^{i}=x^{i}+\left(g^{-1} \mathcal{F}^{-1}(x)\right)^{i k} p_{k} \sigma, \\
2 \pi \alpha^{\prime} P_{i}=p_{i}-p_{m} p_{n} g^{m j} g^{n k}\left(\partial_{j} \mathcal{F}_{k i}(x)\right) \sigma,
\end{gathered}
$$

and

$$
X^{a}=x_{0}^{a}, \quad P_{a}=0,
$$

where we have introduced

$$
p_{k}=\dot{x}^{j} g_{j k}(x) .
$$

The Poisson bracket for the open string is determined by the symplectic two-form ${ }^{1}$

$$
\Omega=\int_{0}^{\pi} d \sigma \delta P_{\mu} \delta X^{\mu}
$$

Substituting Eq. (20) into Eq. (23), we find

$$
\Omega=\frac{1}{2 \alpha^{\prime}} \delta \bar{p}_{i} \delta \bar{x}^{i}
$$

up to second order terms. Here $\bar{p}_{i}$ and $\bar{x}^{i}$ are defined as

$$
\begin{aligned}
& \bar{p}_{i}=p_{i}-\frac{\pi}{2} p_{m} p_{n} g^{m j} g^{n k}\left(\partial_{j} \mathcal{F}_{k i}(x)\right), \\
& \bar{x}^{i}=x^{i}+\frac{\pi}{2}\left(g^{-1} \mathcal{F} g^{-1}(x)\right)^{i k} p_{k} .
\end{aligned}
$$

We thus obtain the commutation relations ${ }^{2}$

$$
\begin{gathered}
{\left[\bar{p}_{i}, \bar{p}_{j}\right]=0, \quad\left[\bar{x}^{i}, \bar{x}^{j}\right]=0,} \\
{\left[\bar{x}^{i}, \bar{p}_{j}\right]=2 i \alpha^{\prime} \delta_{j}^{i},}
\end{gathered}
$$

or equivalently, in terms of $x^{i}$ and $p_{j}$, it is

$$
\begin{gathered}
{\left[p_{i}, p_{j}\right]=0} \\
{\left[x^{i}, x^{j}\right]=2 \pi i \alpha^{\prime}\left(g^{-1} \mathcal{F} g^{-1}\right)^{i j},}
\end{gathered}
$$

\footnotetext{
${ }^{1} \mathrm{We}$ do not need to do the time average prescription here as in [10]; and if we do it here we will obtain the same result.

${ }^{2}$ Normally, special attention has to be paid to the operator ordering when we derive commutation relations from Poisson brackets, so that the Jacobi identity is satisfied if possible. Fortunately, in the approximation we use here, the issue of operator ordering does not arise.
}

$\left[x^{i}, p_{j}\right]=2 i \alpha^{\prime} \delta_{j}^{i}+i \pi \alpha^{\prime} p_{k} g^{k m} g^{i n}\left(\partial_{m} \mathcal{F}_{n j}+\partial_{n} \mathcal{F}_{m j}-\partial_{j} \mathcal{F}_{m n}\right)$.

All of these hold only up to second order terms. It is easy to verify that the commutation relations (29)-(31) satisfy the Jacobi identity also up to second order terms.

It is now easy to check that for $\sigma=0, \pi$,

$$
\left[X^{i}, X^{j}\right]= \pm 2 \pi i \alpha^{\prime}\left(g^{-1} \mathcal{F} g^{-1}\right)^{i j},
$$

where the plus (minus) sign corresponds to the endpoint $\sigma$ $=0(\pi)$. This agrees with the result for a constant background (14) in the leading order. Furthermore, since the right hand side of Eq. (32) is a tensor field, this equation is covariant under general coordinate transformations up to second order terms.

In the static gauge $X^{i}$ is the worldvolume coordinate for the D-brane, so the D-brane worldvolume appears to be a noncommutative space. We propose that in the weak field and slow variation approximation we considered, Eq. (32) gives the commutation relations for the D-brane spacetime coordinates in a generic background.

Under an $S$ duality transformation, a D-string is turned into a fundamental string. For a D-string, the noncommutativity is governed by Eq. (32) with the NS-NS background $\mathcal{F}=B-d A$. The commutation relation for a fundamental string in the dual theory is thus

$$
\left[X^{i}, X^{j}\right]= \pm 2 \pi i g_{s}^{\prime} \alpha^{\prime}\left(g^{-1} \mathcal{F}^{\prime} g^{-1}\right)^{i j},
$$

where $g_{s}^{\prime}=1 / g_{s}$ is the dual string coupling and $\mathcal{F}^{\prime}=B^{\prime}$ $-d A^{\prime}$ is the Ramond-Ramond (R-R) counterpart of $\mathcal{F}$ in the dual theory. Since we assumed that $g_{s}$ is small to derive Eq. (32), Eq. (33) is valid only for large $g_{s}^{\prime}$. It would be interesting if one can derive this directly from string theory.

\section{NONCOMMUTATIVE GAUGE THEORY AND UNCERTAINTY RELATIONS}

The noncommutativity Eq. (32) signifies the existence of uncertainty relations on the brane. To give a precise formulation, we first need to explain what we mean by an uncertainty $\delta X$. The suitable framework for discussing uncertainty relation is to employ the language of string field theory [15]. Let $\Psi$ be the normalized wave function for the D-brane and define $\left(\Delta X^{i}\right)^{2}$ by

$$
\left(\Delta X^{i}\right)^{2}=\int[D X(\xi)] \Psi^{\dagger}(X(\xi))\left(X^{i}(\xi)-\overline{X^{i}}\right)^{2} \Psi(X(\xi)),
$$

where

$$
\overline{X^{i}}=\int[D X(\xi)] \Psi^{\dagger}(X(\xi)) X^{i}(\xi) \Psi(X(\xi))
$$

In these equations, $\xi=\left(\xi_{0}, \ldots, \xi_{p}\right)$ denote the worldvolume coordinates of the D-brane. The part $[D X(\xi)]$ of the functional measure denotes an integration over all D-brane con- 
figurations. Obviously these definitions mimic their counterparts in the familiar case of the quantum mechanics for a point particle.

Applying the standard argument for uncertainty principle to Eq. (32) and noticing that as a background, $\mathcal{F}$ is independent of the D-brane wavefunction $\Psi$, we get

$$
\Delta X^{i} \Delta X^{j} \geqslant 2 \pi l_{s}^{2}\left|\mathcal{F}^{i j}\right|
$$

where $\mathcal{F}^{i j}=\left(g^{-1} \mathcal{F} g^{-1}\right)^{i j}$ and $l_{s}^{2}=\alpha^{\prime}$. Note that these first quantized quantities depend on $\Psi$ and the classical backgrounds. The right hand side of Eq. (36) vanishes for a trivial classical background $\mathcal{F}=0$. However, as explained in [10], even for such a classical background, there could be nontrivial uncertainty relations arising from integrating out the quantum fluctuations. On expanding the string field $\Psi$ into the component fields $\mathcal{B}$, the string field path integral becomes an infinite product of path integrals over these fields and the expectation value $\langle\cdot\rangle$ of an operator in string field theory is defined by

$$
\langle\mathcal{O}\rangle=\frac{1}{Z} \int[D \mathcal{B}] e^{-S} \mathcal{O} \text { where } Z=\int[D \mathcal{B}] e^{-S}
$$

For example, $\mathcal{B}$ includes the metric $g$ and the $B$-field. It is thus natural to define the desired uncertainty for $X^{i}$ as

$$
\delta X^{i}=\sqrt{\left\langle\left(\Delta X^{i}\right)^{2}\right\rangle} .
$$

Using Schwarz inequality, we find

$$
\left(\delta X^{i}\right)^{2}\left(\delta X^{j}\right)^{2} \geqslant\left|\left\langle\Delta X^{i} \Delta X^{j}\right\rangle\right|^{2} .
$$

Thus

$$
\delta X^{i} \delta X^{j} \geqslant 2 \pi l_{s}^{2}\left\langle\left|\mathcal{F}^{i j}\right|\right\rangle
$$

and it generally reduces to something of the form

$$
\delta X^{i} \delta X^{j} \geqslant f\left(g_{s}\right) l_{s}^{2},
$$

where $f\left(g_{s}\right)$ is some function of $g_{s}$. We will try to determine $f$ in the weak coupling limit. Notice that this form of uncertainty relation follows more or less from dimensional analysis. The point of the analysis performed above is to give a precise definition of the quantities involved and to show that the right hand side of Eq. (41) is really nonvanishing.

To see how the $g_{s}$ dependence comes in, it is instructive to consider the case of a D-string. The commutation relation for the worldsheet directions reads

$$
\left[X^{0}, X^{1}\right]=2 \pi i l_{s}^{2} \mathcal{F}
$$

where $\mathcal{F}=\mathcal{F}_{01}$ and we have replaced $g_{i j}$ by the flat metric in our approximation. We thus need to evaluate

$$
\frac{1}{Z} \int[D \mathcal{B}] e^{-S}|\mathcal{F}|
$$

For small $g_{s}$, we can use the tree level supergravity (SUGRA) action where $S$ contains a piece

$$
S=\frac{1}{g_{s}^{2} l_{s}^{8}} \int d B * d B+\ldots
$$

It is convenient to go to a gauge in which $\mathcal{F}=B$ and by rescaling

$$
B=g_{s} B^{\prime},
$$

then for the background $B=0$

$$
\begin{aligned}
\langle|\mathcal{F}|\rangle & \simeq \frac{1}{Z_{B}} \int[D B] \exp \left(-\frac{1}{g_{s}^{2} l_{s}^{8}} \int\left(\partial B_{01}\right)^{2}\right)\left|B_{01}\right| \\
& =g_{s}\left\{\frac{1}{Z_{B}^{\prime}} \int\left[D B^{\prime}\right] \exp \left(-\frac{1}{l_{s}^{8}} \int\left(\partial B_{01}^{\prime}\right)^{2}\right)\left|B_{01}^{\prime}\right|\right\},
\end{aligned}
$$

where

$$
\begin{aligned}
& Z_{B}=\int[D B] \exp \left(-\frac{1}{g_{s}^{2} l_{s}^{8}} \int\left(\partial B_{01}\right)^{2}\right), \\
& Z_{B}^{\prime}=\int\left[D B^{\prime}\right] \exp \left(-\frac{1}{l_{s}^{8}} \int\left(\partial B_{01}^{\prime}\right)^{2}\right) .
\end{aligned}
$$

The above term $\{\cdot\}$ in Eq. (46) is independent of $g_{s}$. One can further scale $B_{01}^{\prime}$ by $l_{s}^{4}$ so that Eq. (46) reads $\langle|\mathcal{F}|\rangle$ $\simeq g_{s} l_{s}^{4} I$, where $I$ is a path integral with no apparent dependence on $g_{s}$ or $l_{s}$. Because of the absolute sign in Eq. (46), it is easy to show that $I$ is nonvanishing and is in fact divergent. A momentum cutoff at $\Lambda$ has to be introduced to make sense of $I$ and one finds $I \sim \Lambda^{4}$ and hence

$$
\langle\mathcal{F}\rangle \simeq g_{s} l_{s}^{4} \Lambda^{4}
$$

The natural cutoff here is $\Lambda \sim l_{s}^{-1}$ because we have ignored all stringy corrections of higher order in $\alpha^{\prime}$ in the SUGRA action.

While the derivation above is not completely rigorous, we consider the possibility of extending this result consistently to all other branes and strings via string dualities in the rest of this paper as a supporting evidence for Eq. (48).

In principle, there could also be other sources contributing to the uncertainty other than the NS-NS 2-form gauge field. Here we considered only the massless mode of SUGRA. In an approximation better than Eq. (42), quantum fluctuations of the metric also contribute. One should also take into account string loop effects for a generic $g_{s}$. These can enter in at least two ways: First, Eq. (32) was derived from a single string in the first order approximation; in general, higher order terms and the string loop effects can modify the commutation relations for the D-brane worldvolume coordinates [16]. Second, more precisely one should also use the string loop corrected SUGRA action instead of the tree level one in 
the above derivation. Including all these factors, we expect the uncertainty relation to take the generic form ${ }^{3}$

$$
\delta X^{0} \delta X^{1} \geqslant g_{s} l_{s}^{2}+\cdots,
$$

up to a numerical factor which will be ignored in this paper, and the omitted terms are of higher order in $g_{s}$ and $\alpha^{\prime}$ due to the above-mentioned higher order corrections. There could also be a dependence on $\Psi$ in the higher order corrections. Obviously one can perform the same derivation for a $\mathrm{D} p$-brane with $p>1$ and find the same uncertainty relation between any two directions on the D-brane.

At this point, one may ask a number of questions. For example, is it possible to determine explicitly the higher order corrections in Eq. (49)? How does the above generalize to the case of the other $\mathrm{D} p$-brane? How does the uncertainty relation look like on a $\mathrm{D} p$-brane? What we will do is to find new uncertainty relations by requiring the uncertainty relations to be consistent with the known dualities of string theory. This consistency requirement will be our guiding principle.

\section{WORLDVOLUME UNCERTAINTY RELATIONS}

In this section, we will propose a form of the uncertainty relations for $\mathrm{D} p$-branes which is consistent with dualities of string theory. To strengthen the starting ground for our argument, which leads to the general result, we first consider the cases of D1 and D0-branes in more detail.

\section{A. D1-branes}

From Sec. III, we find that the uncertainty relation for a D-string in the small $g_{s}$ limit in flat spacetime takes the form

$$
\delta X^{0} \delta X^{1} \geqslant g_{s} l_{s}^{2} .
$$

Eq. (50) gives a minimal area for the D1-brane worldsheet.

An independent support for this result can be obtained by S-duality. Under S-duality, a D1-brane is interchanged with a fundamental string, and the string tension interchanged with the D1-brane tension. Thus the uncertainty relation for a fundamental string should be

$$
\delta X^{0} \delta X^{1} \geqslant l_{s}^{2} .
$$

This is in fact what people have suggested based on properties of string scattering amplitudes, worldsheet conformal invariance, and other various arguments [18]. This can also be heuristically argued as follows (first two references of [18]). According to the canonical uncertainty relation in quantum mechanics

$$
\delta E \delta T \geqslant 1
$$

\footnotetext{
${ }^{3}$ Uncertainty relations for the D-brane worldvolume have also been discussed in the context of Liouville string theory [17]. There an uncertainty relation of a form similar to Eq. (49) was found, but with a different dependence $\left(\sqrt{g_{s}}\right.$ instead of $\left.g_{s}\right)$ on the string coupling.
}

(where $T$ should be identified with $X^{0}$ ), if $\delta T$ is small, $\delta E$ will be large. Since $E \sim 1 / \alpha^{\prime}$ times string length, it is associated with a large uncertainty $\delta X^{1}$ in the string length [19]. (For this argument to be more rigorous, we need a virial theorem stating that a certain portion of the energy must be attributed to the potential energy due to string tension.)

\section{B. D0-branes}

The usual uncertainty principle of quantum mechanics (52) implies that

$$
\delta T \geqslant \frac{1}{\delta E} \geqslant \frac{1}{E}
$$

where we assumed that $E \geqslant \delta E$. (This would be the case if $E$ is positive definite.) In the rest frame of the D0-brane, $E$ $=1 /\left(g_{s} l_{s}\right)$ is the mass of a D0-brane, so we find

$$
\delta T \geqslant g_{s} l_{s},
$$

where $T$ is the proper time for the D0-brane worldline.

We can also interpret Eq. (54) as follows. If Eq. (54) is not satisfied, the energy of a D0-brane can be larger than its rest mass and can thus lead to pair productions of D0 and anti-D0-branes. One can then imagine a quantum path in which the created anti-D0-brane annihilates the original D0brane so that the created D0-brane survives as the final D0brane. In such cases the proper time is ill-defined during the process of creation and annihilation.

In [19] it was explained that the short distance behavior of D-branes is described by the low energy physics of open strings ending on the D-branes. For D0-branes two different characteristic scales were found. The first scale is the Planck scale for the 11 dimensional SUGRA, which is $g_{s}^{1 / 3} l_{s}$ in accordance with the duality between $\mathrm{M}$ theory and type IIA theory. Assuming $g_{s}<1$, the Planck scale is smaller than the string scale $l_{s}$ which characterizes the uncertainty relation for fundamental strings. The Planck scale was found as the characteristic scale of the $(0+1)$-dimensional Super Yang-Mills (SYM) theory, which describes the low energy theory of D0-branes. Although the Planck scale is believed to be the minimal scale in 11D SUGRA, it is not the scale of uncertainty relations for D0-branes since a smaller characteristic scale $g_{s} l_{s}$ was found in [19]. It is called the "fine structure" scale, which can be seen only after taking into account the correction of the SYM action by the Dirac-Born-Infeld (DBI) action. Note that no smaller scale was found in the analysis of [19]. Although the fine structure scale was obtained as the characteristic scale in the transverse (spatial) directions, based on our results above, we propose in this paper that the fine structure scale also sets the minimal length on a D0brane worldline as in Eq. (54).

Another support for the claim that Eq. (54) gives the correct uncertainty relation for D0-branes is obtained via $T$ duality. If the D1-brane has the uncertainty relation $\delta T \delta X$ $\geqslant g_{s} l_{s}^{2}$, then for a D1-brane wrapped on a compactified 
circle with radius $R{ }^{4} \delta X$ cannot be larger than $R$, which implies that $\delta T \geqslant g_{s} l_{s}^{2} / R$. By $T$ duality this is interpreted as a dual D0-brane with the uncertainty $\delta T \geqslant g_{s}^{\prime} l_{s}$, where $g_{s}^{\prime}$ $=g_{s} l_{s} / R$ is the string coupling constant in the dual theory. This is exactly what we claimed in Eq. (54).

Incidentally we note that in terms of $\mathrm{M}$ theory, $g_{s} l_{s}$ is the radius of the compactification through which the $\mathrm{M}$ theory is dual to type IIA theory. By compactifying $\mathrm{M}$ theory on a circle smaller than the Planck scale, the smaller scale $g_{s} l_{s}$ is introduced into the compactified M theory. This would not be possible if there were an uncertainty relation like $\delta X$ $\geqslant l_{p}$ in the uncompactified $\mathrm{M}$ theory. On the other hand, this is consistent with the worldvolume uncertainty relations we find for membranes and 5-branes as in Eqs. (62) and (63).

\section{Dp-branes via $T$ duality}

In the above we have seen that $T$ duality can be used to derive uncertainty relation of D0-branes from that of D1branes. Here we will generalize the arguments to all $\mathrm{D} p$-branes.

For simplicity we first consider the case of a flat background. We know that a D-string can be obtained from a D2-brane under $T$ duality. By wrapping a leg of the D2-brane on the circle, one introduces on the D2-brane worldvolume an uncertainty of the order

$$
\delta X^{2} \sim R
$$

since the center of the D-brane can be anywhere on the circle. It is thus natural to guess that the uncertainty relation on a D2-brane will involve a product of uncertainties of the form

$$
\delta X^{0} \delta X^{1} \delta X^{2} \geqslant g_{s} l_{s}^{3}
$$

which is the product of the uncertainty relation (50) for a D1-brane and Eq. (55) in terms of the dual $g_{s}$ and $l_{s}$.

On the other hand, one can also repeat the derivation of D1-brane uncertainty relation in Sec. III for D2-branes. Since $\delta X^{i} \delta X^{j} \geqslant g_{s} l_{s}^{2}+\cdots$ for all $i \neq j, i, j=0,1,2$, one can derive $\delta X^{0} \delta X^{1} \delta X^{2} \geqslant g_{s}^{3 / 2} l_{s}^{3}+\cdots$. This is a weaker condition than Eq. (56). At this moment we do not know how to derive Eq. (56) directly from open strings ending on D2-branes as in the case of D1-branes. We leave this interesting question for future study.

For a $D p$-brane in general, we propose that

$$
\delta X^{0} \delta X^{1} \ldots \delta X^{p} \geqslant g_{s} l_{s}^{p+1}
$$

in flat spacetime. For a $\mathrm{D} p$-brane in curved spacetime, the natural generalization of the uncertainty relation is just

$$
\delta V_{(p+1)} \geqslant g_{s} l_{s}^{p+1},
$$

\footnotetext{
${ }^{4}$ Since we assume that the string couplings are smaller than 1 , the compactification radius needs to satisfy $l_{s} / g_{s}^{\prime}>R>g_{s} l_{s}$.
}

where $\delta V_{(p+1)}$ is the uncertainty of the $\mathrm{D} p$-brane worldvolume, which is the spacetime volume corresponding to $\delta X^{i}$.

One can check that Eq. (57) respects the $T$ duality of string theory. To see this, suppose that we start with a $\mathrm{D} p$-brane in a string theory compactified on a circle of radius $R$ with string coupling $g_{s}$. We take one of the dimensions of the $\mathrm{D} p$-brane, says $X^{p}$, to be wrapped on the circle. Since $\delta X^{p} \sim R$, we get for the $\mathrm{D}(p-1)$-brane,

$$
\delta X^{0} \delta X^{1} \cdots \delta X^{p-1} \geqslant g_{s}^{\prime} l_{s}^{p}
$$

where $g_{s}^{\prime}=g_{s} l_{s} / R$ is the dual string coupling. Thus Eq. (57) is consistent with $T$ duality.

It is interesting to note that Eq. (57) can be derived from Eq. (52) and

$$
\delta E=\mathcal{T} \delta V_{p},
$$

where $\mathcal{T}=1 / g_{s} l_{s}^{p+1}$ is the tension of the $\mathrm{D} p$-brane. Consider an experiment conducted on the $\mathrm{D} p$-brane which is supposed to measure some point-like process. Because of the nonvanishing spacetime uncertainty relation, the region of the process would appear to have a spatial volume uncertainty of order $\delta V_{p}$. The associated uncertainty in energy would have a typical order of (60) and would be consistent with the standard $T-E$ type uncertainty relation (52). However, to really derive Eq. (57) from Eq. (52), one should in principle also include other possible sources of uncertainties (e.g., contributions from the oscillation modes) in Eq. (60). The fact that one may derive Eq. (57) by simply using Eq. (60) suggests that there might be some sort of stringy virial theorem.

\section{Dyonic branes via $S$ duality}

$S$ duality is expected to be an exact symmetry of type IIB string theory. Under an $S$ duality transformation, a $\mathrm{D} p$-brane gets transformed into an $(m, n)$ - $p$-brane for $p=1,5$. For the purpose of explicit illustrations, we will spell out the $(m, n)$-string case in details. $(m, n)-5$-branes can be treated similarly.

Starting with the uncertainty relation for a D-string (50), we can derive the uncertainty relation for a $(m, n)$-string by the $S L(2, \mathbf{Z})$ transformation of $S$ duality. It is

$$
\delta X^{0} \delta X^{1} \geqslant l_{s}^{2} \frac{1}{\sqrt{(m-n \chi)^{2}+\frac{n^{2}}{g_{s}^{2}}}},
$$

where $\chi$ is the axion vacuum expectation value. Notice that the right hand side is just the inverse of the tension of the $(m, n)$-string. In particular, we get Eq. (51) as a special case.

\section{E. M-branes via M/IIB duality}

Just as the D-brane is the endpoint of an open string, one can also learn about the physics of the M5-brane by considering it as the boundary of an M2-brane (see for example [20] for other applications in this direction). It was shown in 
[10] that the M5-brane worldvolume can also become noncommutative in the presence of $\mathcal{F}_{i j k}$, which is a combination of the three-form gauge field and a worldvolume field strength.

Applying similar considerations as in the string case above, we propose the following uncertainty relation for the M2-brane and M5-brane:

$$
\begin{gathered}
\delta X^{0} \delta X^{1} \delta X^{2} \geqslant l_{p}^{3} \text { for M2-brane, } \\
\delta X^{0} \delta X^{1} \cdots \delta X^{5} \geqslant l_{p}^{6} \text { for M5-brane, }
\end{gathered}
$$

where $l_{p}$ is the 11-dimensional Planck length. An uncertainty relation of the same form as Eq. (62) was proposed in [2] as a result of Eq. (51) due to the M/IIA duality, but with a different interpretation, which is analogous to the one they had for Eq. (71).

We now show that Eq. (61) is related to the membrane uncertainty (62) by using the M/IIB duality. It is known $[21,22]$ that IIB string theory can be obtained from compactifying $\mathrm{M}$ theory on a shrinking 2-torus with radii $R_{1}, R_{11}$. A IIB $(m, n)$-string is identified with a membrane wrapped over the $(m, n)$-cycle on the torus with length

$$
L_{(m, n)}=R_{11} \sqrt{\left(m-n \tau_{1}\right)^{2}+n^{2} \tau_{2}^{2}} .
$$

Here $\tau=\tau_{1}+i \tau_{2}$ is the modular parameter of the torus and it is identified $[21,22]$ with the IIB string theory parameters as

$$
\tau_{1}=\chi, \quad \tau_{2}=1 / g_{s} .
$$

Now starting from Eq. (62) and using

$$
\delta X^{2} \sim L_{(m, n)}
$$

for the uncertainty for the membrane direction which is wrapped on a cycle, we immediately obtain Eq. (61). Here we used $l_{s}^{2}=l_{p}^{3} / R_{11}$.

In fact, since our uncertainty relations can be obtained mathematically from Eqs. (52) and (60), the matching of the brane spectra for dual theories implies that the uncertainty relations must be consistent with all dualities. For instance, it is automatically true that the M/IIA duality also relates Eqs. (62) and (63) to Eqs. (51) and (57) for $p=2,4$. Incidentally, the M/IIA duality also gives rise to the uncertainty relation

$$
\delta X^{0} \cdots \delta X^{5} \geqslant g_{s}^{2} l_{s}^{6}
$$

for the NS5-brane.

\section{DISCUSSIONS}

In this paper, we discussed the uncertainty relations for the D-brane worldvolume. We introduced the notion of a worldvolume uncertainty and explained how it is defined within the context of string field theory. We proposed worldvolume uncertainty relations that are consistent with the various dualities in string theory. We have also generalized the commutation relation for the noncommutative gauge theory to a nontrivial background of $\mathcal{F}$ in the lowest order approximation. It would be interesting to generalize this result to the full generality of an arbitrary background. This could be relevant to the interesting proposal in [23]. In the following we remark on several related subjects.

\section{A. Comments on some other uncertainty relations}

\section{Uncertainty relation of Wigner}

In the classical study of Wigner [24], the effects of quantum mechanics on the measurability of the spatial distance were estimated to be given by

$$
\delta D \geqslant \sqrt{T / M_{c}} .
$$

Here $T$ is the time scale for the process involved and $M_{c}$ is the mass of the clock. This analysis has recently been strengthened in [25], which utilizes the existence of a Schwarzschild horizon $R_{s}$ for any massive object and thus it is necessary that

$$
\delta D \geqslant R_{s} \sim G M_{c} .
$$

Combining with Eq. (68), one obtains

$$
(\delta D)^{3} \geqslant G T .
$$

Notice that in this analysis, the precision of the measurement of time is not limited. The uncertainty relation we proposed is consistent with these results. For example, in the brane world scenario, when $\delta T=0$, our uncertainty relation for a D3-brane says that $\delta D=\infty$, which is stronger than Eqs. (68) or (70).

The argument leading to Eq. (70) utilizes the most popular reason for the belief in the existence of spacetime uncertainty relations. That is, due to quantum mechanics a large energy is needed to probe a small length scale, and when the energy is too large a black hole is formed, which forbids the measurement of distances behind the horizon. However, in our derivation of the uncertainty relations (57), we did not mention anything about event horizon at all. It remains to be seen how the consideration of black holes can lead to the determination of uncertainty relations in string theory, and whether it will lead to new uncertainty relations.

\section{Uncertainty relation of Yoneya}

Notice that our uncertainty relations (57) are not of the same type as those proposed in $[2,4,5,26]$. These authors proposed uncertainty relations that involve the transverse coordinates while ours are solely for the brane world. For example, in $[2,4,5]$, it was proposed that

$$
\delta T \delta X \geqslant l_{s}^{2}
$$

for a $\mathrm{D} p$-brane. Here $\delta T$ is understood as the uncertainty in the longitudinal directions on the brane (to be more precise, $\delta T=|\delta \sigma|$, where $\sigma$ is the worldvolume coordinates in the static gauge) and $\delta X$ represents the uncertainty in directions transverse to the brane. As it was pointed out in [19], the short distance regime of D-branes are probed by open strings. The exchange of a closed string state between two D-branes, for example, can be viewed as the creation and 
annihilation of a pair of open strings (an open string loop diagram) due to the modular invariance of string theory. Therefore, the scattering of D-branes is limited by the uncertainty of open strings, and Eq. (71) is a direct result of Eq. (51).

Our uncertainty relation (57), on the other hand, is concerned with the uncertainty among longitudinal directions on the brane, and it has a form different from Eq. (71). In particular ours have a form that depends on the dimensionality of the brane and have additional dependence on $g_{s}$.

\section{B. UV-IR relations, holography and uncertainty relations}

In $[5,2]$, a generalized conformal symmetry was found for the $\mathrm{D} p$-brane super Yang-Mills action. It is easy to check that the $(p+1)$-dimensional YM action with coupling $g_{\mathrm{YM}}$, which is schematically

$$
S=\frac{1}{g_{\mathrm{YM}}^{2}} \int d^{p+1} \sigma\left((\partial X)^{2}+X^{4}\right),
$$

is invariant under the following scaling transformation

$$
\begin{gathered}
X^{a} \rightarrow \lambda X^{a}, \quad \sigma_{i} \rightarrow \lambda^{-1} \sigma_{i}, \\
g_{\mathrm{YM}}^{2} \rightarrow \lambda^{3-p} g_{\mathrm{YM}}^{2} .
\end{gathered}
$$

Here $X^{a}(a=p+1, \ldots, 9)$ are the transverse scalars and $\sigma_{i}(i=0,1, \ldots p)$ are the worldvolume coordinates in the static gauge. The coupling $g_{\text {YM }}^{2}$ is related to the string coupling by

$$
g_{\mathrm{YM}}^{2}=g_{s} l_{s}^{p-3} .
$$

The uncertainty relation (71) is invariant under this scaling (73) together with $g_{s} \rightarrow \lambda^{3-p} g_{s}$ and $l_{s}$ being invariant.

Notice that Eq. (73) is reminiscent of the UV-IR distance relation $[27,28]$ in the context of AdS conformal field theory (CFT) holography [29-31]. Maybe this scaling relation is a generic property for general holographic dualities $[32,33]$. It was discussed in $[2,34]$ that the uncertainty relation (71) is consistent with the UV-IR relation and it was suggested that the uncertainty principle is the underlying principle that implies the UV-IR relation, which in turn guarantees that the holographic bound [33] will be satisfied [27].

Since our uncertainty relations are only involved with the worldvolume uncertainties, the scaling (73) and (74) does not give any nontrivial implications on our relations. On the other hand, since our uncertainty relation implies the existence of a minimal area, it may also be relevant to the holographic principle and to the verification of the holographic bound. It would be interesting if one could see this explicitly. Another interesting issue is that based on the UV-IR relation and holographic principle, it is natural to ask what kind of spacetime property (presumably a spacetime uncertainty relation) will be implied by the worldvolume uncertainty relations. We leave these issues for future studies.

\section{Characteristic scale}

The scaling transformation (73) and (74) can also be used to find the characteristic scale of YM theory. Let $\lambda$ $=g_{s}^{1 /(p-3)}$, then we scale $g_{s} \rightarrow 1$ and $X \rightarrow g_{s}^{1 /(p-3)} X$. This means that the Higgs vacuum expectation value $g_{s}^{1 /(p-3)} X$ is independent of $g_{s}$, and thus the YM characteristic scale is $g_{s}^{-1 /(p-3)} l_{s}$. For $p=0$ this gives the Planck scale $g_{s}^{1 / 3} l_{s}$. For $p=3$ it is the string scale $l_{s}$. For $p>3$ this scale is much larger than the string scale, but for these cases YM theory is not renormalizable and it means that we cannot trust it.

As mentioned in Sec. IV B, a characteristic scale does not have to be the minimal scale. For the case of D0-branes, it is the characteristic scale of the DBI action that turns out to be the minimal scale. It is thus of interest to also work out the characteristic scale for the DBI theory for a $\mathrm{D} p$-brane. The DBI action is

$$
S=\frac{1}{g_{s} l_{s}^{p+1}} \int d^{p+1} \sigma \sqrt{-\operatorname{det}(g+\mathcal{F})},
$$

which is invariant under the scaling

$$
X \rightarrow \lambda X, \quad g_{s} \rightarrow \lambda^{p+1} g_{s},
$$

and an arbitrary scaling of $\sigma$. Letting $\lambda=g_{s}^{-1 /(p+1)}$, we find $g_{s} \rightarrow 1$ and $X \rightarrow g_{s}^{-1 /(p+1)} X$. This means that $g_{s}^{1 /(p+1)} l_{s}$ is the characteristic scale of the DBI action for the transverse directions. It happens that this characteristic scale is also the one determining the minimal volume in our uncertainty relations (58). It is possible that this is also the minimal length scale for the transverse directions of a $\mathrm{D} p$-brane much like the case of D0-branes discussed in [19].

Even if both the transverse and longitudinal directions of a D0-brane are bounded by this scale $g_{s} l_{s}$, Eq. (71) is still a stronger condition than just the product of the two minimal lengths. In Sec. IV C we also mentioned that Eq. (57) is a stronger condition than a product of Eq. (50) for each pair of longitudinal directions. It seems that in string theory we need a complicated web of uncertainty relations, which cannot be deduced from a single master relation, to fully state the uncertainty property of spacetime.

\section{ACKNOWLEDGMENTS}

C.S.C. thanks G. Amelino-Camelia and A. Bilal for helpful discussions. He is also grateful to the Department of Physics and the Center for Theoretical Physics at the National Taiwan University for hospitality where part of this work was carried out. P.M.H. and Y.C.K. thank S. Das and particularly M. Li for helpful discussions. P.M.H. thanks M. M. Sheikh-Jabbari for helpful remarks. The work of C.S.C. is supported by the Swiss National Science Foundation. The work of P.M.H. and Y.C.K. is supported in part by the National Science Council (NSC 88-2112-M-002-042, NSC 882112-M-002-034) and the Center for Theoretical Physics, National Taiwan University, Taiwan, Republic of China. 
[1] M. R. Douglas, LXIV Les Houches Session on Quantum Symmetries, 1995, hep-th/9610041.

[2] M. Li and T. Yoneya, "Short-Distance Space-Time Structure and Black Holes in String Theory,"' hep-th/9806240.

[3] G. Venezario, Europhys. Lett. 2, 199 (1986); D. Gross and P. Mende, Nucl. Phys. B303, 407 (1988); D. Amati, M. Ciafaloni, and G. Veneziano, Phys. Lett. B 216, 41 (1989); M. Fabbrichesi and G. Veneziano, ibid. 233, 135 (1989); K. Konishi, G. Paffuti, and P. Provero, ibid. 234, 276 (1990).

[4] T. Yoneya, Prog. Theor. Phys. 97, 949 (1997); “D-Particles, D-Instantons, and Space-Time Uncertainty Principle in String Theory," hep-th/9707002.

[5] A. Jevicki and T. Yoneya, Nucl. Phys. B535, 335 (1998); A. Jevicki, Y. Kazama, and T. Yoneya, Phys. Rev. D 59, 066001 (1999).

[6] See, for example, N. Arkani-Hamed, S. Dimopoulos, G. Dvali, Phys. Rev. D 59, 086004 (1999); Z. Kakushadze and S.-H. H. Tye, Nucl. Phys. B548, 180 (1999).

[7] A. Connes, M. R. Douglas, and A. Schwarz, J. High Energy Phys. 02, 003 (1998).

[8] M. R. Douglas and C. Hull, J. High Energy Phys. 2, 8 (1998).

[9] M. M. Sheikh-Jabbari, Phys. Lett. B 425, 48 (1998).

[10] C. S. Chu and P. M. Ho, Nucl. Phys. B550, 151 (1999).

[11] Y.-K. E. Cheung and M. Krogh, Nucl. Phys. B528, 185 (1998); T. Kawano and K. Okuyama, Phys. Lett. B 433, 29 (1998); P.-M. Ho, ibid. 434, 41 (1998). See also [12].

[12] F. Ardalan, H. Arfaei, and M. M. Sheikh-Jabbari, "Mixed Branes and M(atrix) Theory on Noncommutative Torus," hep-th/9803067; J. High Energy Phys. 02, 016 (1999).

[13] C. Hofman and E. Verlinde, J. High Energy Phys. 12, 010 (1998).

[14] H. Garcia-Compean, Nucl. Phys. B541, 651 (1999).

[15] See, for example, M. B. Green, J. H. Schwarz, and E. Witten, Superstring Theory (Cambridge University Press, Cambridge, England, 1987); J. Polchinski, String Theory (Cambridge University Press, Cambridge, England, 1998).

[16] V. Schomerus, J. High Energy Phys. 06, 030 (1999).
[17] G. Amelino-Camelia, J. Ellis, N. E. Mavromatos, and D. V. Nanopoulos, Mod. Phys. Lett. A 12, 2029 (1997).

[18] T. Yoneya, in Wandering in the Fields, edited by K. Kawarabayashi and A. Ukawa (World Scientific, Singapore, 1987), p. 419; Mod. Phys. Lett. A 4, 1587 (1989); M. Li and T. Yoneya, Phys. Rev. Lett. 78, 1219 (1997).

[19] M. R. Douglas, D. Kabat, P. Pouliot, and S. H. Shenker, Nucl. Phys. B485, 85 (1997).

[20] C. S. Chu and E. Sezgin, J. High Energy Phys. 9712, 001 (1997); C. S. Chu, P. S. Howe, and E. Sezgin, Phys. Lett. B 428, 59 (1998); C. S. Chu, P. S. Howe, E. Sezgin, and P. C. West, ibid. 429, 273 (1998).

[21] J. H. Schwarz, Phys. Lett. B 360, 13 (1995); 364, 252(E) (1995).

[22] P. S. Aspinwall, Nucl. Phys. B (Proc. Suppl.) 46, 30 (1996).

[23] A. Jevicki and S. Ramgoolam, J. High Energy Phys. 04, 032 (1999).

[24] E. P. Wigner, Rev. Mod. Phys. 29, 255 (1957).

[25] N. Sasakura, "An Uncertainty Relation of Spacetime," hep-th/9903146.

[26] N. E. Mavromatos and R. J. Szabo, Phys. Rev. D 59, 064016 (1999); 59, 104018 (1999); "D-Branes and the NonCommutative Structure of Quantum Spacetime,', hep-th/9811116.

[27] L. Susskind and E. Witten, "The Holographic Bound in Anti-de Sitter Space,', hep-th/9805114.

[28] C. S. Chu, P. M. Ho, and Y. Y. Wu, Nucl. Phys. B541, 179 (1999); M. Bianchi, M. B. Green, S. Kovacs, and G. Rossi, J. High Energy Phys. 08, 013 (1998); A. W. Peet and J. Polchinski, Phys. Rev. D 59, 065011 (1999).

[29] J. Maldacena, Adv. Theor. Math. Phys. 2, 231 (1998).

[30] S. S. Gubser, I. R. Klebanov, and A. M. Polyakov, Phys. Lett. B 428, 105 (1998).

[31] E. Witten, Adv. Theor. Math. Phys. 2, 253 (1998).

[32] G. 't Hooft, "Dimensional Reduction in Quantum Gravity," gr-qc/9310006.

[33] L. Susskind, J. Math. Phys. 36, 6377 (1995).

[34] D. Minic, Phys. Lett. B 442, 102 (1998). 